\title{
BMJ Open Development of automated quantification methodologies of immunohistochemical markers to determine patterns of immune response in breast cancer: a retrospective cohort study
}

Carlos López, ${ }^{1}$ Cristina Callau, ${ }^{2}$ Ramon Bosch, ${ }^{3}$ Anna Korzynska, ${ }^{4}$ Joaquín Jaén, ${ }^{3}$ Marcial García-Rojo, ${ }^{5}$ Gloria Bueno, ${ }^{6}$ Ma Teresa Salvadó, ${ }^{3}$ Tomás Álvaro, ${ }^{3}$ Montse Oños, ${ }^{7}$ María del Milagro Fernández-Carrobles, ${ }^{6}$ Montserrat Llobera, ${ }^{8}$ Jordi Baucells, ${ }^{9}$ Guifré Orero, ${ }^{2}$ Marylène Lejeune ${ }^{2}$

To cite: López C, Callau C, Bosch R, et al. Development of automated quantification methodologies of immunohistochemical markers to determine patterns of immune response in breast cancer: a retrospective cohort study. BMJ Open 2014:4:e005643. doi:10.1136/bmjopen-2014005643

- Prepublication history for this paper is available online. To view these files please visit the journal online (http://dx.doi.org/10.1136/ bmjopen-2014-005643).

Received 7 May 2014 Accepted 11 July 2014

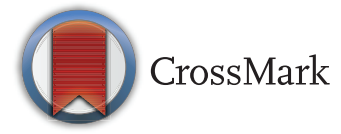

For numbered affiliations see end of article.

Correspondence to Dr Carlos López; clpclp3@gmail.com

\section{ABSTRACT}

Introduction: Lymph nodes are one of the main sites where an effective immune response develops.

Normally, axillary nodes are the first place where breast cancer produces metastases. Several studies have demonstrated the importance of immune cells, especially dendritic cells, in the evolution of breast cancer. The goal of the project is to identify differences in the patterns of immune infiltrates, with particular emphasis on dendritic cells, in tumour and axillary node biopsies between patients with and without metastases in the axillary nodes at the time of diagnosis. It is expected that these differences will be able to explain differences in survival, relapse and clinicopathological variables between the two groups. Methods and analysis: The study will involve 100 patients diagnosed with invasive breast cancer between 2000 and $2007,50 \%$ of whom have metastases in the axillary lymph node at diagnosis. In selected patients, two cylinders from biopsies of representative areas of tumour and axillary nodes (with and without metastasis) will be selected and organised in tissue microarrays. Samples will be stained using immunohistochemical techniques for different markers of immune response and dendritic cells. Two images of each cylinder will be captured under standardised conditions for each marker. Each marker will be quantified automatically by digital image procedures using Image-Pro Plus and Image-J software. Associations of survival, relapse and other clinicopathological variables with the automatically quantified levels of immune infiltrates in patients with and without axillary node metastasis will be sought.

Ethics and dissemination: The present project has been approved by the Clinical Research Ethics Committee of the Hospital Universitari Joan XXIII (Ref: 22p/2011). Those patients whose biopsies and clinical data are to be used will give their signed informed consent. Results will be published in peer-reviewed journals.

\section{Strengths and limitations of this study}

- Normally, a percentage of images cannot be analysed by automated methods because of poor staining. We have noticed that this happens more frequently in very old samples (pre-2000) or with immunohistochemical stains that are not widely used. However, in this project, the samples have been obtained after 2000 and most of the markers involved are widely used in pathology departments. Under these conditions we would expect that a relatively low percentage of images would not be amenable to analysis.

- Image analysis procedures for immune infiltrate evaluation in tumour tissue give a high reproducibility in evaluations and allow large numbers of markers and samples to be quantified under similar conditions.

- At the same time this study will evaluate the immune infiltrate in breast cancer tumours and in axillary lymph nodes of previously diagnosed patients with and without metastasis since most previous studies have evaluated these areas or these groups of patients separately.

\section{INTRODUCTION}

Breast cancer is the main cause of mortality from malignant tumours in women in western developed nations. In recent years, several strategies and therapies have been developed to cure patients or prolong their survival. However, some of these therapies have side effects and, in patients with distal metastasis, rarely have a curative effect. ${ }^{1}$ Several strategies have recently been used to develop treatments that are more effective and less harmful to patients. Therapeutic 
vaccine is one such strategy that is showing particular promise for the future. These vaccines induce the immune system to detect and eliminate exclusively tumoral cells without causing collateral damage. ${ }^{2}$

Dendritic cell (DC) vaccines are being studied in several tumours and in breast cancer. ${ }^{2}{ }^{3}$ The results obtained so far have not been completely satisfactory. ${ }^{4}$ DCs are the most important antigen-presenting cells (APCs). They originate in the bone marrow then move into the bloodstream and migrate to the peripheral tissues. At that point DCs are immature and carry out sentinel functions, waiting for signals-normally infection or damage that promotes inflammation-that trigger their maturation. During this period DCs are very effective at catching and processing protein antigens from the environment, but remain relatively inactive as APCs. At the appropriate time they migrate into the lymphatic vessels and travel to the regional lymph nodes, where they mature and present the antigens to $\mathrm{T}$ lymphocytes and activate the immune response. The main functions of DCs are: (1) to capture antigens and migrate to the lymphoid organs to optimise the clonal selection of CD4 and CD8 T cells; (2) to promote immune response-stimulating quiescent, and $\mathrm{T}$ and B memory lymphocytes; (3) to improve the T cellmediated immune response; (4) to induce tolerance to the body's own antigens. ${ }^{3}$

Most DCs belong to a myeloid lineage and others to a lymphoid lineage. Those of the latter type are also known as plasmacytoid DCs. The various subpopulations of myeloid DCs (HLA'DR ${ }^{+} \mathrm{CD} 11 \mathrm{c}^{+}$) can express markers such as CD1a (Langerhans cells), CD21 (follicular DCs), S-100 (interdigitating cells), while the plasmacytoid DCs express CD123. Immature DCs do not express a significant quantity of costimulator molecules such as CD80, CD86 or CD40 at their surface, and produce very small quantities of IL-12, a cytokine essential for establishing a mediated immune response in Th1 cells, and one of great importance in antitumour immunity. When DCs mature they start to express markers like CD83 and DC-Lamp. DCs have several functions and stages of maturity, and depending on the lineage and stage, they express different types of molecules. ${ }^{5}$

Previous studies of breast cancer have shown that the presence of $\mathrm{CD}^{+} \mathrm{a}^{+}$DCs is correlated with a better prognosis and evolution of patients. ${ }^{6}$ Similar studies of DCs in patients with a high tumoral burden have demonstrated that activating the DCs administrating (granulocyte macrophage colony-stimulating factor) can prolong the survival of patients by improving the antitumour effect. ${ }^{7}$ A higher concentration of $\mathrm{S}-100^{+}$DCs has been observed in grade III invasive breast cancer than in grade I and II patients. ${ }^{8}$ The presence of these S- $100^{+}$ DCs is correlated with tumour size, affectation of axillary lymph nodes, oestrogen and progesterone receptor status and age. However, the overall survival (OS) and disease-free survival (DFS) of these patients is not influenced by the presence of S- $100^{+}$DCs. ${ }^{8}$ On the other hand, one study has demonstrated that infiltration of CD123 plasmacytoid cells is associated with an unfavourable evolution, suggesting that they contribute to tumour progression in breast cancer. ${ }^{9}$ The same study confirmed that mature CD208/DC-LAMP ${ }^{+}$DCs are positively correlated with the presence of lymph node with metastasis and with tumoral grade.

Tumour infiltration by mature CD83 ${ }^{+}$DCs could be of great importance in initiating the primary immune response against the tumour. The converse of the case for $\mathrm{CD}_{1 \mathrm{a}}{ }^{+}$immature DCs, the frequency of these mature CD83 DCs is inversely correlated with the presence of axillary nodes with metastasis, the level of expression of vascular endothelial growth factor and transforming growth factor- $\beta$ in the tumour, and they have been confirmed as being an immunologically independent prognostic factor for survival in patients with breast cancer. ${ }^{10}$ Other markers of maturity, such as CMRF-44 and CMRF-56, are deficient in breast cancer, irrespective of the histological grade of the tumour. ${ }^{11}$

Since part of the DC immune response occurs in lymph nodes, where antigens captured in the exterior are presented to lymphocytes, it is very important to evaluate the immune response at this location as well as in the tumour. Greater infiltration of mature $\mathrm{CD} 83^{+}$DCs has been observed in sentinel nodes without tumours than in those invaded by tumours. ${ }^{12}$ Thus, tumour-free sentinel nodes are immunologically competent and a potential site for the activation of specific T cells. On the other hand, the immune response dependent on DC-Lamp DCs is altered during metastasis formation, and is activated before and during the formation of micrometastases. ${ }^{13}$

Nevertheless, DCs are involved in only one of the first steps in the antitumour immune response. If this step is altered, the subsequent immune response may not be appropriate. Thus, it is also necessary to evaluate what happens with the general immune response at the tumour and lymph node levels.

The recruitment of immune and/or inflammatory cells in invasive breast cancer is one of the procedures involved in the antitumour immune response. Studies have demonstrated the presence of a heterogeneous population of immune cells in the tumour microenvironment of the breast. Basically, this population comprises $\mathrm{T}$ lymphocytes (CD4, CD8), ${ }^{14}$ cytotoxic cells (Tcell intracellular antigen-1 (TIA)-1, granzyme $\mathrm{B}),{ }^{15}$ tumour-associated macrophages (CD68), ${ }^{14}$ DCs (CD21, $\mathrm{CD} 1 \mathrm{a}, \mathrm{CD} 123)^{9}$ and regulatory $\mathrm{T}$ cells (FOXP3). ${ }^{16} \mathrm{In}$ some of these studies, the type and amount of these immune cells have been linked to clinical variables such as tumour size, age, disease progression and patient survival. ${ }^{17}$ The importance of these cellular infiltrates has also been used to predict clinicopathological performance. ${ }^{9}{ }^{14}$ Moreover, the evaluation of components of the immune response from tumour and stroma could help differentiate the different types and stages of the illness. ${ }^{18}$ The presence of cells with immunosuppressive capacity in the tumour (regulatory cells), ${ }^{19}$ as well as 
tolerance induction by tumour cells, ${ }^{20}$ could explain why some tumours grow and metastasise progressively. A significant difference in the number of regulatory $\mathrm{T}$ cells has been detected between human epidermal growth factor receptor 2 (HER2)-positive and HER2-negative patients with advanced breast cancer. ${ }^{21}$

In breast cancer, most research has partially addressed the subpopulations of immune cells present in the tumour or in the lymph nodes. Moreover, most of the cited articles have investigated only the tumour or the lymph nodes. Thus, it will be of interest to determine the global pattern of the immune response (DCs and other immune cells) simultaneously in breast cancer tumours and axillary lymph nodes to detect any differences associated with tumour evolution.

Most immune markers can be evaluated using immunohistochemistry (IHC), with which several cell subpopulations can be detected and quantified in situ in the tumour or lymph node. IHC is simpler, cheaper and faster than other molecular biological techniques. The markers can be evaluated quickly and efficiently through the use of tissue microarrays (TMAs), the standardisation of stain conditions of immunohistochemical markers, and the detection and quantification of markers by automated digital image analysis. ${ }^{22}$ Digital analysis can minimise the problems of human subjectivity between evaluators. ${ }^{23}$ In recent years, the use of digital image analysis of immunohistochemical markers in breast cancer has become important. Several systems, such as Automated Cellular Imaging System (ACIS) III (DAKO, Carpinteria), have been developed and commercialised and subsequently approved by the US Food \& Drug Administration. ${ }^{24}$ Nevertheless, these systems only allow some types of immunohistochemical markers to be quantified, such as the membrane marker CerbB2, and nuclear markers, such as oestrogen and progesterone receptors.

The aims of this aspect of the proposed project are: (1) to develop new image analysis methods to quantify immune markers arising from other project activities; (2) to search for different patterns of immune response cells and specifically of DC infiltrates in the tumour and axillary lymph nodes, comparing patients with and without metastasis in the axillary lymph nodes; (3) to identify any associations between immune infiltrates and clinicopathological variables and patient survival.

\section{METHODS AND ANALYSIS}

\section{Study design and setting}

The study will examine a retrospective cohort of 100 patients with invasive breast cancer diagnosed in Hospital de Tortosa Verge de la Cinta, Catalonia, Spain, between 2000 and 2007. Fifty per cent of the patients had metastasis in the axillary lymph nodes at diagnosis. The amount and localisation of different types of immune infiltrates, especially of DCs, in tumour and in axillary lymph nodes will be evaluated in the patient biopsies. Immune infiltrates will be detected and evaluated using IHC and image analysis procedures. Differences in the patterns of immune infiltrates will be sought in the tumour biopsies and in axillary lymph nodes with and without metastases between the two groups of selected patients (with and without metastasis in axillary lymph nodes at diagnosis). Associations of survival, relapse and other clinicopathological variables with the immune infiltrate patterns will be sought.

\section{Study period}

The study will be undertaken between January 2012 and December 2014.

\section{Study sample}

The study will involve only the samples that fulfil the following inclusion criteria: invasive breast cancer samples with enough representative tumour (more than $50 \%$ of selected area must contain tumour) and axillary lymph node tissue in patient biopsies. Of these, only samples from patients who have given signed informed consent will be used.

Samples collected from 2000 to 2007 will be selected from the Tumour Bank of the Pathology Department of the Hospital de Tortosa Verge de la Cinta, as part of the XBTC included in the BioBanc node HTVC - Biobanc Hospital Universitari Joan 23 - IISPV. This period has been chosen to ensure enough samples with at least 5 years of follow-up in both patient groups studied (with and without axillary lymph node metastases).

Tumour and axillary node paraffin-embedded biopsies will be used to study the immune infiltrate in patients with breast cancer. In those without metastasis in axillary lymph nodes at diagnosis, two cylinders of $2 \mathrm{~mm}$ each from representative areas of the tumour and two from axillary lymph nodes will be taken. In patients with metastasis, six cylinders will be evaluated, two from the tumour, two from metastasis-free axillary nodes and two from axillary nodes with metastasis.

\section{Sample size calculation}

The study includes 100 patients, based on the sample size and power calculations for identifying relative risk available at http://www.imim.es/ofertadeserveis/softwarepublic/granmo/. The calculation was performed to find an immune infiltrate pattern able to predict a death risk in the group with metastasis 1.6 times that of the group without metastases. Values used in the calculation are described below.

Accepting an $\alpha$ risk of 0.05 and a $\beta$ risk of 0.2 in a twosided test, 50 participants exposed to certain expected type of immune pattern and 50 non-exposed participants are necessary for a relative risk of death greater than or equal to 1.6 in the exposed group to be statistically significant. The proportion of deaths among the people in the non-exposed group has been estimated to be 0.5 , based on the principle of maximum indeterminacy, since there are no previous data about the type of pattern of immune infiltrate that can predict death. A drop-out rate of $10 \%$ has been anticipated. 


\section{Samples and data processing}

1. TMA construction: Twenty TMAs of 25 cylinders extracted from biopsies will be constructed. Eight TMAs will be constructed from tumour biopsies ( 2 cylinders/patient $) \times 100$ patients $) /(25$ cylinders/ TMAs). Eight TMAs will be obtained from axillary lymph nodes free of metastasis, and four will be from axillary lymph nodes with metastasis since only $50 \%$ of patients belong to this group.

2. Slide preparation: Eleven immune and DC markers will be evaluated by IHC. For this purpose, 11 sections of $2 \mu \mathrm{m}$ thickness will be cut from each TMA to stain all markers. A total of 220 slides will be stained (20 TMAs $\times 11$ markers).

3. Clinical immune markers studied

A. Markers for general immune response detection: CD4 (usually expressed in T helper lymphocytes), CD8 (usually expressed in cytotoxic T lymphocytes), CD57 (expressed in Natural Killer lymphocytes), FOXP3 (expressed in regulatory $\mathrm{T}$ cells), CD21 (expressed in mature B lymphocytes), CD68 (expressed on the surface of macrophages).

B. Markers for DC detection: CD1a (dendritic Langerhans cells), CD123 (plasmacytoid DCs), S100 (interdigitating DCs) CD208 (DC Lamp ${ }^{+}$), CD83 (marker of mature DCs).

4. IHC staining of samples: slides will be stained fully automatically with the Autostainer Link 48 (DAKO) from the pathology department. The use of TMAs allows automation and the staining of a large number of samples quickly and under the same conditions. Quality controls for breast cancer used in the pathology department for daily clinical practice will be stained at the same time as the samples of the study. In this way, the positive and negative controls as well as the quality of the counterstaining may be compared with the IHC staining of the study samples. Moreover, all the TMAs will be stained for the same markers in the same time in order to ensure the same conditions of staining (same antibody dilution, same temperature, etc.).

5. Standardisation of image capture: To obtain maximum reproducibility in image analysis the conditions of image capture will be standardised for each marker, which means that we will have the same illumination conditions and a standard white balance.

Two images from the area with the most abundant cellular infiltration of each cylinder will be captured, so by the end of this step we will have 11000 digital images: ((200 tumoral cylinders+200 axillary lymph node without metastasis cylinder +100 axillary lymph node with metastasis cylinder $) \times(2$ pictures $) \times(11$ markers $)$. As seen in our previous studies, two images taken from a tumour area have been demonstrative enough for immune cell infiltrate evaluation. ${ }^{5}{ }^{7}$ Tissue sections will be viewed using brightfield illumination under a Leica DM LB2 upright light microscope (Leica Microsystems Wetzlar GmbH,
Wetzlar, Germany) with a $40 \times$ plane-apochromatic objective with a numerical aperture of 0.63 . The representative areas of the different sections will be captured on a Leica DFC320 digital camera (Leica Microsystems Digital Imaging, Cambridge, UK) controlled by Leica DFC Twain software V.6.3.0 (Leica Microsystems Digital Imaging) running on a Compaq Professional Workstation computer (3.6 GHz Pentium IV CPU, 3.00 GB RAM). These images will have a resolution of $2088 \times 1550$ pixels with RGB 24 true colour format, and will be saved in uncompressed tagged-image file format (TIFF). The same range of illumination values will be used to allow maximum reproducibility to avoid differences in the illumination, since they can produce significant differences in the results of image quantification.

6. Development of image analysis procedures: Image analysis procedures for quantifying the 11 immunohistochemical markers will be adapted for each marker from our automated image analysis procedures developed in previous studies (see point 7 ). These methods will be validated by comparing the results obtained from several manual (pathologist) evaluations. Several strategies and image analysis techniques will be used:

- Light image correction.

- Determination of the most suitable space colour model (RGB, HSI, CMYK, etc.) for analysing each marker, and of colour or grey scale ranges for positive and negative objects.

- Use of morphological filters in case they are needed to increase the efficiency of detection algorithms.

- Detection of nuclear or cell edges.

- Division of cell clusters in the images, if necessary.

- Mathematical correction or extrapolation, if necessary.

- Development of some algorithms by collaborating centres (University of Castilla La Mancha, Spain, and the Nalecz Institute of Biocybernetics and Biomedical Engineering, Warsaw, Poland).

7. Automated image analysis: The automated procedures developed in the present project will be based on automated procedures developed in our previous studies. The main features to take into account for the development of new procedures will be stain localisation (nuclear, membrane or cytoplasmic), ${ }^{25} 26$ and the presence of background and its stain intensity in image. ${ }^{27}$ In these studies the development of the image analysis procedures will be explained step-by-step. For the evaluation of the new markers included in the present project, the previous automated methods will be adapted using the image analysis techniques previously described in the part 6. Despite the large number of images produced (11 000), it will not take very long to analyse them. The image analysis procedures developed for each marker will be run consecutively, the analysis of 50 images taking approximately $20 \mathrm{~min}$. 
8. Compilation of patient data: Clinical and pathological data from patients enrolled in the study will be collected from electronic and paper medical records during the first year of the project. Clinical information related to the amount and localisation of DCs and other immune cells infiltrating the tumour and axillary lymph nodes will be collected during the second part of the third year of the project after quantifying them with image analysis procedures. Survival and relapse data will be collected during the last trimester of the study in order to have the longest follow-up. The date from last follow-up will be the date of the most recent visit to the hospital or primary care centre.

9. Analysis of results: The results will be of two types.

A. Validation of image analysis procedures: Differences, reproducibility and concordance between manual and automated methods will be evaluated by the intraclass correlation coefficient, Student's t test, Bland-Altman and Kaplan-Meier graphs and ROC curves.

B. Immune response patterns: The evaluation of immune response of each case will be based on the mean of the evaluation of a total of four images: two images taken from each of the two cylinders of each case. The cases where one cylinder is lost due to the cut of samples or a bad immunostain will be discarded from the analysis. We have estimated a $10 \%$ of samples loss that have been included in the sample size calculation mentioned above.

Illness progression (relapse or not) and survival function (alive or dead) based on the immune infiltrates and the other clinicopathological variables will be evaluated by the Kaplan-Meier method and Cox regression to evaluate the association between the various markers and progression/survival of illness. The methodology for evaluating the relation of immune infiltrates with cancer progression on patient's survival has been previously tested in our group's previous studies. ${ }^{5} 728$ The statistics methods for this evaluation are described below.

The Kaplan-Meier method for estimating DFS and OS will be used. Two groups will always be compared using several cut-off points in the number of cells or in the level of molecular marker expression: mean, first quartile, second quartile, third quartile or the presence or absence of stained molecular markers.

The concentrations of cells and molecular markers will be compared between patients with and without metastasis. They will be analysed by the $\chi^{2}$ test when grouped as categories and by the Student $t$ test when evaluated quantitatively. The same type of analysis will be performed for clinical variables depending on whether they are quantitative or categorical.

A Cox regression model will be fitted to compute the HR and its 95\% CIs relapse/survival with each biomarker in the study. Those biomarkers with significance under 0.10 will be included in a multivariable Cox
Regression model to obtain a relapse prediction model. Those clinical variables that could influence patients' relapse will also be included in the multivariable Cox Regression model. Statistical analysis will be performed with the software IBM SPSS Statistics V.21.0.

\section{Study limitations}

Normally, a percentage of images cannot be analysed by automated methods because of poor staining. We have noticed that this happens more frequently in very old (pre-2000) samples or with immunohistochemical stains that are not widely used. However, in this project, the samples will have been obtained after 2000 and most of the markers involved are widely used in pathology departments. Under these conditions we would expect that a relatively low percentage of images would prove not possible to analyse.

\section{ETHICS AND DISSEMINATION}

Patients whose biopsies and clinical data will be used will provide signed informed consent. Results will be published in peer-reviewed journals.

The study guarantees the confidentiality of patients' data. The data collected and generated will be included in two databases as a data-decoupling procedure to ensure adherence to data protection legislation and to maintain individual privacy. For this reason, different types of files will be created and maintained, each with a different password. Passwords will be changed periodically and will only be known to the person in charge.

The first file will be a demographic database in which each patient is assigned a unique code. The second file will include the patient codes linked to the information relevant to the study, including the results of the study and clinical variables for statistical analysis. The databases will remain stored on the renewably passwordprotected hard disk of a PC located in a locked office with controlled access. Results will be published in peerreviewed journals and disseminated in international conferences and meetings related to digital pathology, immunology or cancer. It is expected that at least three articles will be published, two of which will be cover image analysis procedures and the third will present the clinical findings related to immune infiltrates and patient survival and relapse.

\section{DISCUSSION}

To understand cancer initiation and progression, it is necessary to focus on the cancer cell and on the tumour microenvironment from which the cancer cell obtains its nutrients, oxygen and signals for growth and development. ${ }^{29}$ The concept of the tumour microenvironment, developed from Paget's 'seed and soil' theory, describes a dynamic network that includes the cancer cells, stromal tissue and the extracellular matrix that surrounds them. 
Immune cells are an important class of stromal cells within the tumour microenvironment. Very few studies have evaluated the immune response in tumours and in lymph nodes simultaneously in the same patients. Moreover, with the development of more accurate methods for analysing immune infiltrates, it is becoming clearer that different infiltrating cell types have distinct prognostic and predictive significance. ${ }^{30}{ }^{31}$ Several studies have demonstrated the presence of a heterogeneous population of immune cells in the breast tumour microenvironment, consisting mainly of $\mathrm{T}$ lymphocytes, ${ }^{14}$ cytotoxic cells, ${ }^{15}$ tumour-associated macrophages, ${ }^{14}$ DCs $^{9}$ and regulatory cells. ${ }^{16}$ The types and amounts of these infiltrated immune cells are significantly associated with some clinical variables, such as tumour size, age, disease progression and survival,,${ }^{17}$ as well as the clinicopathological behaviour of the patient. ${ }^{9}{ }^{14}$ Moreover, more than just the composition, distribution, architecture and functional articulation of the immune infiltrate, the immune context must be analysed to understand its significance and to derive more accurate prognostic and predictive biomarkers. ${ }^{32}$ This may involve analysing and evaluating the composition of the immune cells in the axillary lymph nodes.

A better understanding of the immune response, in particular that of DCs in patients with breast cancer, may help improve the design and application of therapeutic vaccines, making them more effective. In breast cancer, immunotherapy has been shown to prevent relapse, to improve survival and eliminate breast cancer in humans. ${ }^{33}$ Immunotherapy fits within the definition of personalised treatment, which is one of the two key technologies that is transforming pathology. The second of these technologies is digital pathology. ${ }^{34}$ Although the validation of these digital approaches has reaffirmed the accuracy of pathologists and the semiquantitative scoring methods they use, ${ }^{24}$ these new methods potentially require less pathologist time and resources, and produce a more objective assessment. There are two additional technologies that allow the in situ evaluation of a large number of molecular markers in a more rapid and homogeneous manner. The first is IHC, a cheap and accessible diagnostic technique used in daily clinical practice in pathology departments. Antibodies used in IHC are among the most frequently used tools in modern biomedical research, their popularity being reflected in the large number of immunohistochemical studies carried out over the past 20 years. ${ }^{35}$ The second is the TMA, which has become an established and valuable tool in cancer research, particularly in translational research and clinical trials. It allows resource-efficient use and high-throughput profiling of large numbers of tumours. The use of TMAs to assess biomarkers has become significantly more common over the past few years. Although the degree of correlation between TMAs and whole-tissue sections may not be considered ideal at the diagnostic level for individual patients, it is widely regarded as adequate in the research setting, where large samples are available for clinical trials or investigative studies. ${ }^{36}$

In the present project, we expect that the use of TMAs and digital image analysis will enable us to identify differences in the patterns of immune response cells and specifically of DC infiltrates in the tumour and axillary lymph nodes between patients with and without metastasis in the axillary lymph nodes. We envisage being able to use this information not only as a source of prognostic factors but also to help improve the design and application of therapeutic vaccines, thereby making them more effective.

\section{Author affiliations}

${ }^{1}$ Unitat de Suport a la Recerca Terres de l'Ebre, IISPV, Institut Universitari d'Investigació en Atenció Primària (IDIAP) Jordi Gol, URV, UAB, Tortosa, Spain

${ }^{2}$ Molecular Biology and Research Section, Hospital de Tortosa Verge de la Cinta, IISPV, URV, Tortosa, Spain

${ }^{3}$ Department of Pathology, Hospital de Tortosa Verge de la Cinta, IISPV, Tortosa, Spain

${ }^{4}$ Laboratory of Processing Systems of Microscopic Image Information, Nalecz Institute of Biocybernetics and Biomedical Engineering, Polish Academy of Sciences, Warsaw, Poland

${ }^{5}$ Department of Pathology, Hospital de Jerez, Jerez de la Frontera, Cadiz, Spain

${ }^{6}$ VISILAB, Engineering School, Universidad de Castilla-La Mancha, Ciudad Real, Spain

${ }^{7}$ Department of Gynaecology, Hospital de Tortosa Verge de la Cinta, IISPV, Tortosa, Spain

${ }^{8}$ Department of Oncology, Hospital de Tortosa Verge de la Cinta, IISPV, Tortosa, Spain

${ }^{9}$ Department of Informatics, Hospital de Tortosa Verge de la Cinta, IISPV, Tortosa, Spain

Acknowledgements The authors thank María del Mar Barbera, Bárbara Tomás, Vanesa Gestí, Ainhoa Montserrat, Ana Suñé and Marc Iniesta for their skilful technical assistance, and Anna Carot and Rosa Cabrera for their excellent secretarial work.

Contributors $\mathrm{CL}$ as principal investigator, and $\mathrm{ML}$ as senior researcher, were involved in the conception of the project, the study design and drafting the manuscript. RB, JJ and TA evaluated and revised the patient biopsies and selected the cylinders for TMA construction. $\mathrm{CL}, \mathrm{MO}$ and MoL compiled the pathological and clinical data of all patients enrolled in the study and created the database. AK, JB, GB and MG-R were involved in standardising image capture conditions and developing the image analysis procedures. MdMF-C, CC and GO captured images and applied image analysis procedures to them. $\mathrm{CL}, \mathrm{ML}$ and MTS developed the final database and statistically analysed the results. CL drafted the first version of the manuscript. All authors read and approved the final version of the manuscript.

Funding This work was supported by grant number PI11/0488 from the Instituto de Salud Carlos III, Spain. Institute of Health Carlos III is the main Public Research Entity funding, managing and carrying out biomedical research in Spain. This Institute reports directly to the Ministry of Economy and Competitiveness (Royal Decree 345/2012) and in operational terms to this Ministry and to the Ministry of Health, Social Services and Equality (Royal Decree 200/2012).

Competing interests $\mathrm{CL}, \mathrm{RB}, \mathrm{JJ}, \mathrm{MTS}, \mathrm{TA}, \mathrm{MoL}, \mathrm{JB}$ and $\mathrm{ML}$ are members of the Oncological Pathology and Bioinformatics Research Group of the Hospital de Tortosa Verge de la Cinta (Pere Virgili Health Research Institute, Spain). CC is a PhD student associated with this study and GO works in the Molecular Biology and Research section of the Hospital de Tortosa. MO was a gynaecologist at the Hospital de Tortosa. AK is an engineer at the Nalecz Institute of Biocybernetics and Biomedical Engineering, Warsaw, Poland. MG-R is a specialist in telepathology at Hospital de Jerez, Spain. GB and 
MdMF-C are members of the Machine Vision and Intelligence Systems (VISILAB) group at the Engineering School of the Universidad de Castilla-La Mancha, Spain.

Patient consent Obtained.

Ethics approval The present project was evaluated by the Clinical Research Ethics Committee of the Hospital Universitari Joan XXIII, Tarragona, Spain, on 28 September 2011 (Ref: 22p/2011).

Provenance and peer review Not commissioned; externally peer reviewed.

Open Access This is an Open Access article distributed in accordance with the Creative Commons Attribution Non Commercial (CC BY-NC 4.0) license, which permits others to distribute, remix, adapt, build upon this work noncommercially, and license their derivative works on different terms, provided the original work is properly cited and the use is non-commercial. See: http:// creativecommons.org/licenses/by-nc/4.0/

\section{REFERENCES}

1. Barrett SV. Breast cancer. J R Coll Physicians Edinb 2010;40:335-8; quiz 39.

2. Soliman H. Developing an effective breast cancer vaccine. Cancer Control 2010;17:183-90.

3. Pinzon-Charry A, Schmidt C, Lopez JA. Dendritic cell immunotherapy for breast cancer. Expert Opin Biol Ther 2006;6:591-604.

4. Koski GK, Cohen PA, Roses RE, et al. Reengineering dendritic cell-based anti-cancer vaccines. Immunol Rev 2008;222:256-76.

5. Alvaro T, Lejeune M, Salvado MT, et al. Immunohistochemical patterns of reactive microenvironment are associated with clinicobiologic behavior in follicular lymphoma patients. J Clin Oncol 2006;24:5350-7.

6. La Rocca G, Anzalone R, Corrao S, et al. CD1a down-regulation in primary invasive ductal breast carcinoma may predict regional lymph node invasion and patient outcome. Histopathology 2008;52:203-12.

7. Alvaro T, Lejeune M, Garcia JF, et al. Tumor-infiltrated immune response correlates with alterations in the apoptotic and cell cycle pathways in Hodgkin and Reed-Sternberg cells. Clin Cancer Res 2008;14:685-91.

8. Lespagnard L, Gancberg D, Rouas G, et al. Tumor-infiltrating dendritic cells in adenocarcinomas of the breast: a study of 143 neoplasms with a correlation to usual prognostic factors and to clinical outcome. Int J Cancer 1999;84:309-14.

9. Treilleux I, Blay JY, Bendriss-Vermare N, et al. Dendritic cell infiltration and prognosis of early stage breast cancer. Clin Cancer Res 2004;10:7466-74.

10. Iwamoto $M$, Shinohara $H$, Miyamoto $A$, et al. Prognostic value of tumor-infiltrating dendritic cells expressing CD83 in human breast carcinomas. Int J Cancer 2003;104:92-7.

11. Coventry BJ, Lee PL, Gibbs D, et al. Dendritic cell density and activation status in human breast cancer-CD1a, CMRF-44, CMRF-56 and CD-83 expression. Br J Cancer 2002;86:546-51.

12. Poindexter NJ, Sahin A, Hunt KK, et al. Analysis of dendritic cells in tumor-free and tumor-containing sentinel lymph nodes from patients with breast cancer. Breast Cancer Res 2004:6:R408-15.

13. Bembenek A, Li J, Loddenkemper C, et al. Presence of mature DC-Lamp+ dendritic cells in sentinel and non-sentinel lymph nodes of breast cancer patients. Eur J Surg Oncol 2008;34:514-18.

14. Macchetti AH, Marana HR, Silva JS, et al. Tumor-infiltrating CD4+ T lymphocytes in early breast cancer reflect lymph node involvement. Clinics (Sao Paulo) 2006;61:203-8.

15. Hussein MR, Hassan HI. Analysis of the mononuclear inflammatory cell infiltrate in the normal breast, benign proliferative breast disease, in situ and infiltrating ductal breast carcinomas: preliminary observations. J Clin Pathol 2006;59:972-7.
16. Triebel F, Hacene K, Pichon MF. A soluble lymphocyte activation gene-3 (sLAG-3) protein as a prognostic factor in human breast cancer expressing estrogen or progesterone receptors. Cancer Lett 2006;235:147-53.

17. Hadden JW. The immunology and immunotherapy of breast cancer: an update. Int $\mathrm{J}$ Immunopharmacol 1999;21:79-101.

18. Lopez C, Lejeune M, Bosch R, et al. Digital image analysis in breast cancer: an example of an automated methodology and the effects of image compression. Stud Health Technol Inform 2012;179:155-71.

19. Liyanage UK, Moore TT, Joo HG, et al. Prevalence of regulatory T cells is increased in peripheral blood and tumor microenvironment of patients with pancreas or breast adenocarcinoma. J Immunol 2002;169:2756-61.

20. Folgueras AR, Pendas AM, Sanchez LM, et al. Matrix metalloproteinases in cancer: from new functions to improved inhibition strategies. Int J Dev Biol 2004;48:411-24.

21. Perez SA, Karamouzis MV, Skarlos DV, et al. CD4+CD25+ regulatory T-cell frequency in HER-2/neu (HER)-positive and HER-negative advanced-stage breast cancer patients. Clin Cancer Res 2007;13:2714-21.

22. Bolton KL, Garcia-Closas M, Pfeiffer RM, et al. Assessment of automated image analysis of breast cancer tissue microarrays for epidemiologic studies. Cancer Epidemiol Biomarkers Prev 2010;19:992-9.

23. Gavrielides MA, Gallas $B D$, Lenz $P$, et al. Observer variability in the interpretation of HER2/neu immunohistochemical expression with unaided and computer-aided digital microscopy. Arch Pathol Lab Med 2011;135:233-42.

24. Rojo MG, Bueno G, Slodkowska J. Review of imaging solutions for integrated quantitative immunohistochemistry in the Pathology daily practice. Folia Histochem Cytobiol 2009;47:349-54.

25. Lopez C, Lejeune M, Salvado MT, et al. Automated quantification of nuclear immunohistochemical markers with different complexity. Histochem Cell Biol 2008;129:379-87.

26. Lejeune M, Jaen J, Pons L, et al. Quantification of diverse subcellular immunohistochemical markers with clinicobiological relevancies: validation of a new computer-assisted image analysis procedure. J Anat 2008;212:868-78.

27. Lejeune M, Gesti V, Tomas B, et al. A multistep image analysis method to increase automated identification efficiency in immunohistochemical nuclear markers with a high background level. Diagn Pathol 2013;8:S13.

28. Alvaro T, Lejeune M, Camacho FI, et al. The presence of STAT1-positive tumor-associated macrophages and their relation to outcome in patients with follicular lymphoma. Haematologica 2006;91:1605-12.

29. Weber CE, Kuo PC. The tumor microenvironment. Surg Oncol 2012;21:172-7.

30. Fridman $\mathrm{WH}$, Galon J, Pages F, et al. Prognostic and predictive impact of intra- and peritumoral immune infiltrates. Cancer Res 2011;71:5601-5.

31. Fridman WH, Pages F, Sautes-Fridman C, et al. The immune contexture in human tumours: impact on clinical outcome. Nat Rev Cancer 2012;12:298-306.

32. Pages F, Galon J, Dieu-Nosjean MC, et al. Immune infiltration in human tumors: a prognostic factor that should not be ignored. Oncogene 2010;29:1093-102.

33. Wright SE. Immunotherapy of breast cancer. Expert Opin Biol Ther 2012;12:479-90.

34. Slodkowska J, Garcia-Rojo M. Digital pathology in personalized cancer therapy. Stud Health Technol Inform 2012;179:143-54.

35. Kalyuzhny AE. The dark side of the immunohistochemical moon: industry. J Histochem Cytochem 2009;57:1099-101.

36. Pinder SE, Brown JP, Gillett C, et al. The manufacture and assessment of tissue microarrays: suggestions and criteria for analysis, with breast cancer as an example. J Clin Pathol 2013;66:169-77. 